Proof. If $M$ is Ricci flat it is trivially Einstein and, since $L^{2}-H L$ $=0$, one of the principal curvatures is zero.

If $R^{*}=b I$, then $L^{2}-H L+b I=0$. If $K=0$, then there is a zero principal curvature and a unit principal vector $X$ with $L X=0$. Hence $b X=0$ so $R^{*}=0$.

In the case $n=3$, the characteristic polynomial $L^{8}-H L^{2}+J L-K I$ $=0$ implies $J L=0$, and since $L_{m}=0$ implies $J(m)=0$, we have $J \equiv 0$.

\title{
BIBLIOGRAPHY
}

1. S. Goldberg, Curvature and homology, Academic Press, New York, 1962.

2. S. Helgason, Differential geometry and symmetric spaces, Academic Press, New York, 1962.

3. N. Hicks, Connexion preserving, conformal, and parallel maps, Michigan Math. J. 10 (1963), 295-302.

4. - Submanifolds of semi-Riemannian manifolds, Rend. Circ. Mat. Palermo 12 (1964), 1-13.

UNIVERSITY OF MichigAN

\section{ON PSEUDOMETRICS FOR GENERALIZED UNIFORM STRUCTURES}

\section{SOLOMON LEADER}

In [1] Alfsen and $\mathrm{Njåstad} \mathrm{generalized} \mathrm{the} \mathrm{concept} \mathrm{of} \mathrm{a} \mathrm{uniform}$ structure $\mathcal{U}$ on a set $S$, replacing the intersection axiom for uniform structures by the weaker condition:

(0) Given subsets $A_{1}, \cdots, A_{n}$ of $S$ and $U_{1}, \cdots, U_{n}$ in $\mathcal{u}$, there exists $U$ in $u$ such that $U\left(A_{i}\right) \subseteq U_{i}\left(A_{i}\right)$ for $i=1, \cdots, n$. Our object is to characterize these structures in terms of pseudometrics.

Define a (generalized) gage on $S$ to be a nonvoid family $\mathcal{G}$ of pseudometrics on $S \times S$ such that

(1) Every pseudometric uniformly continuous with respect to $\mathcal{G}$ belongs to $\mathrm{g}$.

(2) If $\alpha$ and $\beta$ belong to $\mathcal{G}$ and both $\alpha$ and $\beta$ are totally bounded, then $\alpha \bigvee \beta$ belongs to $\mathrm{g}$.

Note that if we delete the total boundedness condition in (2), then $\mathcal{G}$ is just a gage for a proper uniform structure [2], [3]. For $\beta$ a pseudometric on $S \times S$, define $W_{\beta}=\beta^{-1}[0,1)$.

TheOREM. Given a gage $\mathcal{G}$ on $S$, define the class $\mathcal{U}$ of subsets $U$ of $S \times S$ by the condition

Received by the editors January 28, 1964. 
(i) $U \in \mathcal{U}$ iff $U \supseteq W_{\beta}$ for some $\beta$ in $\mathrm{g}$.

Then $\mathfrak{u}$ is a generalized uniform structure for which

(ii) $\alpha \in \mathcal{G}$ iff $W_{n \alpha} \in \mathcal{U}$ for every positive integer $n$.

Conversely, given a generalized uniform structure $u$ on $S$, define the family $G$ of pseudometrics $\alpha$ by (ii). Then $G$ is a gage for which (i) holds.

Lemma. Given a pseudometric $\alpha$ on $S \times S$ and nonempty subsets $A$ and $B$ of $S$ such that $\alpha(A, B) \geqq 1$, there exists a totally bounded pseudometric $\beta$ such that $\beta \leqq \alpha$ and $\beta(A, B)=1$.

To prove the lemma, let $f(s)=\alpha(s, A)[\alpha(s, A)+\alpha(s, B)]^{-1}$ on $S$ and define $\beta(x, y)=|f(x)-f(y)|$. A few simple computations show that $\beta$ has the desired properties.

To prove the theorem, let $\mathcal{G}$ satisfy (1) and (2) and define $\mathcal{u}$ by (i). That $\mathfrak{u}$ has all the properties of a uniform structure except for the intersection axiom follows exactly as in the case of proper uniform structures. To prove (0) we may, in view of (i), assume that $U_{i}=W_{\alpha}$ for some $\alpha_{i}=\alpha$ in $g$. Let $B_{i}=S \backsim U_{i}\left(A_{i}\right)$. Since the conclusion of (0) will be trivial wherever $A_{i}$ or $B_{i}$ is empty, we may assume both are nonempty. Apply the lemma to get $\beta_{i}$ totally bounded with $\beta_{i} \leqq \alpha_{i}$ and $\beta_{i}\left(A_{i}, B_{i}\right)=1$. $\beta_{i}$ is in $\mathcal{G}$ by (1). Let $\beta=\beta_{1} \vee \cdots \vee \beta_{n}$, which is in $\mathcal{G}$ by (2). Let $U=W_{\beta}$. Given $y$ in $U\left(A_{i}\right),(x, y)$ is in $U$ for some $x$ in $A_{i}$. That is, $\beta_{i}(x, y) \leqq \beta(x, y)<1$ for some $x$ in $A_{i}$. So $\beta_{i}\left(y, A_{i}\right)<1$. Since $\beta_{i}\left(A_{i}, B_{i}\right)=1, y$ is not in $B_{i}$. That is, $y$ is in $U_{i}\left(A_{i}\right)$. Thus (0) holds and $\mathcal{u}$ is a generalized uniform structure.

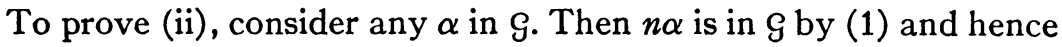
$W_{n \alpha}$ is in $\mathcal{u}$ by (i). Conversely, let $W_{n \alpha}$ belong to $\mathcal{u}$ for all $n$. By (i) there exists for each $n$ some $\beta$ in $\mathcal{S}$ such that $W_{\beta} \subseteq W_{n \alpha}$. Thus (1) implies $\alpha$ is in $\mathcal{G}$.

Given a generalized uniform structure $\mathcal{U}$, define $\mathcal{G}$ by (ii). We must prove (1), (2), and (i). For $\beta$ uniformly continuous relative to $\mathcal{G}$ and $m$ any positive in teger, there exist $\alpha$ in $G$ and a positive integer $n$ such that $W_{n \alpha} \subseteq W_{m \beta}$. Since $W_{n \alpha}$ belongs to $\mathcal{u}$ by (ii), so does $W_{m \beta}$. So $\beta$ is in $\mathcal{G}$ by (ii). Hence (1) holds just as in the case of proper uniform structures.

To prove (2) let $\alpha$ and $\beta$ be totally bounded members of $\mathcal{G}$. Let $\gamma=\alpha \bigvee \beta$. Since $\gamma$ is totally bounded, we can get a finite covering $S_{1} \cup \ldots \cup S_{k}=S$ with diameters $\gamma\left[S_{i}\right]<1 / 4$. Applying $(0)$ to the sequences

$$
\left\{\begin{array}{c}
S_{1}, \cdots, S_{k}, S_{1}, \cdots, S_{k} \\
W_{2 \alpha}, \cdots, W_{2 \alpha}, W_{2 \beta}, \cdots, W_{2 \beta}
\end{array}\right\}
$$

we get $U$ in $u$ such that 


$$
U\left(S_{i}\right) \subseteq W_{2 \alpha}\left(S_{i}\right) \cap W_{2 \beta}\left(S_{i}\right) \text { for } i=1, \cdots, k .
$$

Consider any $(x, y)$ in $U$. Since $x$ is in some $S_{i}, y$ is in the corresponding $U\left(S_{i}\right)$. Hence (3) implies $\gamma\left(y, S_{i}\right)<3 / 4$. So $\gamma(x, y) \leqq \gamma\left(x, S_{i}\right)$ $+\gamma\left[S_{i}\right]+\gamma\left(y, S_{i}\right)<0+1 / 4+3 / 4=1$. That is, $U \subseteq W_{\gamma}$. So $W_{\gamma}$ belongs to $\mathcal{U}$ whenever $\alpha$ and $\beta$ are totally bounded members of $\mathcal{G}$. Using (1) we can apply this result to $n \alpha$ and $n \beta$ to conclude that $W_{n \gamma}$ belongs to $\mathcal{~}$. That is, $\gamma$ is in $\mathcal{G}$. So (2) holds.

To prove (i) let $U$ be any member of $\mathcal{u}$. Choose a sequence $\left\{U_{n}\right\}$ in $\mathcal{u}$ such that $U_{n}=U_{n}^{-1}$ and $U_{n+1}^{3} \subseteq U_{n} \subseteq U$ for all $n$. By the Metrization Lemma [3] there exists a pseudometric $\beta$ such that

$$
U_{n+1} \subseteq W_{2^{n-1} \beta} \subseteq U_{n} \quad \text { for all } n .
$$

$\beta$ is in $\mathcal{G}$ by (4) and (ii). Setting $n=1$ in (4) yields $W_{\beta} \subseteq U$ which proves the direct implication in (i). The converse follows from (ii) since $W_{\beta}$ is in $\mathcal{U}$ if $\beta$ is in $g$.

Using the lemma and [4], we obtain the following corollaries.

COROLLARY 1. For a given proximity relation, let $\mathrm{I}$ be the associated precompact gage and $\mathrm{g}$ be the associated total [1] gage. Then $\mathrm{g}$ consists of all pseudometrics $\alpha$ on $S \times S$ such that every totally bounded pseudometric $\beta$ satisfying $\beta \leqq \alpha$ belongs to $\Im$.

Corollary 2. A gage $g$ is total iff $g$ contains every pseudometric $\alpha$ for which every totally bounded pseudometric $\beta$ satisfying $\beta \leqq \alpha$ belongs to $\mathrm{g}$.

\section{REFERENCES}

1. E. M. Alfsen and O. Njåstad, Proximity and generalized uniformity, Fund. Math. 52 (1963), 235-252.

2. L. Gillman and M. Jerison, Rings of continuous functions, Van Nostrand, Princeton, N. J., 1960.

3. J. L. Kelley, General topology, Van Nostrand, Princeton, N. J., 1955.

4. S. Leader, On a problem of Alfsen and Fenstad, Math. Scand. 13 (1963), 44-46.

Rutgers, The State University 